\title{
Convexity and concavity of the complete elliptic integrals with respect to Lehmer mean
}

\section{Yu-Ming Chu ${ }^{1 *}$ and Tie-Hong Zhao ${ }^{2}$}

${ }^{*}$ Correspondence:

chuyuming2005@126.com

'School of Mathematics and Computation Sciences, Hunan City

University, Yiyang, 413000, China

Full list of author information is

available at the end of the article

\begin{abstract}
In this paper, the authors present necessary and sufficient conditions for the complete elliptic integrals of the first and second kind to be convex or concave with respect to the Lehmer mean.
\end{abstract}

MSC: $33 \mathrm{C05} ; 26 \mathrm{E} 60$

Keywords: complete elliptic integral; generalized convexity; Lehmer mean

\section{Introduction and main results}

\subsection{Legendre's complete elliptic integrals}

For $r \in[0,1]$, Legendre's complete elliptic integrals of the first and second kind $[1,2]$ are defined by

$$
\mathcal{K}(r)=\int_{0}^{\pi / 2}\left(1-r^{2} \sin ^{2} \theta\right)^{-1 / 2} d \theta
$$

and

$$
\mathcal{E}(r)=\int_{0}^{\pi / 2}\left(1-r^{2} \sin ^{2} \theta\right)^{1 / 2} d \theta,
$$

respectively. Note that $\mathcal{K}(0)=\mathcal{E}(0)=\pi / 2$ and $\mathcal{K}(1)=\infty, \mathcal{E}(1)=1$.

It is well known that the complete elliptic integrals have many important applications in geometric function theory, theory of mean values, number theory, and many other areas of mathematics, as well as physics and engineering [3-9].

Because of the importance of the complete elliptic integrals, they have been studied extensively by many researchers from different points. The asymptotic behavior of $\mathcal{K}(r)$ near the singularity $r=1$ has been explored by Kühnau [10], and Alzer and Qiu [11]. Many remarkable inequalities and monotonicity properties for $\mathcal{K}(r)$ and $\mathcal{E}(r)$ can be found in the literature $[4,12-23]$. The generalization of the complete elliptic integrals was first introduced by Vuorinen et al. in [24], and subsequently they were studied intensively in [25-29].

\subsection{Generalized convexity}

In order to introduce the generalized convexity, we first recall the definition of mean function and several classical means.

(c) 2015 Chu and Zhao. This article is distributed under the terms of the Creative Commons Attribution 4.0 International License (http://creativecommons.org/licenses/by/4.0/), which permits unrestricted use, distribution, and reproduction in any medium, provided you give appropriate credit to the original author(s) and the source, provide a link to the Creative Commons license, and indicate if changes were made. 
Definition 1.1 ([14], Definition 2.1) A function $M:(0, \infty) \times(0, \infty) \rightarrow(0, \infty)$ is said to be a mean function if

(1) $\min (x, y) \leq M(x, y) \leq \max (x, y)$,

(2) $M(x, x)=x$

(3) $M(x, y)=M(y, x)$,

(4) $M(a x, a y)=a M(x, y)$ for all $a>0$.

\section{Example 1.2}

(1) $A(x, y)=(x+y) / 2$ is the arithmetic mean;

(2) $G(x, y)=\sqrt{x y}$ is the geometric mean;

(3) $H(x, y)=2 x y /(x+y)$ is the harmonic mean;

(4) $H_{p}(x, y)=\left[\left(x^{p}+y^{p}\right) / 2\right]^{1 / p}$ if $p \neq 0$, and $H_{0}(x, y)=G(x, y)=\sqrt{x y}$ is the $p$ th Hölder (power) mean;

(5) $L_{p}(x, y)=\left(x^{p+1}+y^{p+1}\right) /\left(x^{p}+y^{p}\right)$ is the $p$ th Lehmer mean.

Definition 1.3 ([14], Definition 2.3) Let $I$ be a subinterval of $(0, \infty), f: I \rightarrow(0, \infty)$ be a continuous function, and $M$ and $N$ be any two mean functions. Then $f$ is said to be $(M, N)$-convex (concave) on $I$ if

$$
f(M(x, y)) \leq(\geq) N(f(x), f(y))
$$

for all $x, y \in I$. Moreover, if the inequality (1.3) is strict except for $x=y$, then $f$ is said to be strictly $(M, N)$-convex (concave) on $I$.

In particular, if both $M$ and $N$ are Hölder means, then (1.3) reduces to

$$
f\left(H_{p}(x, y)\right) \leq(\geq) H_{q}(f(x), f(y)),
$$

and $f$ is said to be $H_{p, q}$-convex (concave) on $I$. Also, if inequality (1.4) is strict except for $x=y$, then $f$ is said to be strictly $H_{p, q}$-convex (concave) on $I$.

Recently, the generalized convexity or concavity has attracted the attention of many mathematicians [30-40]. Baricz [30] proved that $\mathcal{K}(r)$ is strictly $H_{p, p}$-convex on $(0,1)$ for $p \in(0,2]$. Zhang et al. [39] improved Baricz's result and proved that $\mathcal{K}(r)$ is strictly $H_{p, q^{-}}$ convex on $(0,1)$ for $(p, q) \in\{(p, q) \mid p \leq 2, q \geq 0\}$. In [41], the authors presented the least value $p_{1}$ and greatest value $p_{2}$ such that $H_{p}(\mathcal{K}(x), \mathcal{K}(y)) \geq \mathcal{K}\left(H_{p}(x, y)\right)$ or $H_{p}(\mathcal{E}(x), \mathcal{E}(y)) \leq$ $\mathcal{E}\left(H_{p}(x, y)\right)$ for all $p \in\left[p_{1}, p_{2}\right]$ and $x, y \in(0,1)$. Very recently, the $H_{p, q^{-}}$convexity and $H_{p, q^{-}}$ concavity of the complete elliptic integrals are discussed in $[33,38]$.

Theorem 1.4 ([38], Theorem 1.4) Let

$$
C(q)=\inf _{r \in(0,1)}\left\{(q-1)\left(\mathcal{E}-r^{\prime 2} \mathcal{K}\right) /\left(r^{\prime 2} \mathcal{K}\right)+r^{2}\left(2 \mathcal{E}-r^{\prime 2} \mathcal{K}\right) /\left[r^{\prime 2}\left(\mathcal{E}-r^{\prime 2} \mathcal{K}\right)\right]\right\}
$$

be a continuous function with $C(q)=2$ for all $q \geq-7 / 2$ and $C(q)<2$ for all $q<-7 / 2$. Then the complete elliptic integrals of the first kind $\mathcal{K}(r)$ is strictly $H_{p, q}$-convex if and only if

$$
(p, q) \in D=\{(p, q) \mid p \leq C(q)\}
$$

and there are no values of $p$ and $q$ for which $\mathcal{K}(r)$ is $H_{p, q}$-concave on $(0,1)$. 
Theorem 1.5 ([33], Theorem 1.5) Let

$$
D(q)=\inf _{r \in(0,1)}\left\{r^{2} \mathcal{E} /\left[r^{\prime 2}(\mathcal{K}-\mathcal{E})\right]+(1-q)(\mathcal{K}-\mathcal{E}) / \mathcal{E}\right\}
$$

be a continuous function with $D(q)=2$ for all $q \leq 5 / 2$ and $D(q)<2$ for all $q>5 / 2$. Then the complete elliptic integrals of the second kind $\mathcal{E}(r)$ is strictly $H_{p, q}$-concave if and only if

$$
(p, q) \in D^{*}=\{(p, q) \mid p \leq D(q)\}
$$

and there are no values of $p$ and $q$ for which $\mathcal{E}(r)$ is $H_{p, q}$-convex on $(0,1)$.

The main purpose of this short note is to establish the necessary and sufficient conditions for the convexity or concavity of the complete elliptic integrals of the first and second kind with respect to the Lehmer mean. Our main results are as follows.

Theorem 1.6 The complete elliptic integral of the first kind $\mathcal{K}(r)$ is strictly $L_{\lambda, \lambda}$-convex on $(0,1)$ if and only if $\lambda \in[-1,0]$.

Theorem 1.7 The complete elliptic integral of the second kind $\mathcal{E}(r)$ is strictly $L_{\lambda, \lambda}$-concave on $(0,1)$ if and only if $\lambda \in(-\infty, 0]$.

\section{A lemma}

In order to prove our main results we need a lemma, which we present in this section.

Lemma 2.1 (see [42]) The inequality

$$
H_{2 t+1}(x, y) \leq L_{t}(x, y)
$$

holds for all $x, y \in \mathbb{R}^{+}$if $t \in(-1,-1 / 2) \cup(0,+\infty)$, and the inequality

$$
H_{2 t+1}(x, y) \geq L_{t}(x, y)
$$

holds for all $x, y \in \mathbb{R}^{+}$ift $\in(-\infty,-1) \cup(-1 / 2,0)$. Inequality (2.1) or (2.2) becomes an equality for all $x, y \in \mathbb{R}^{+}$if $t=-1,-1 / 2$, or 0 ; otherwise inequality (2.1) or (2.2) becomes an equality only when $x=y$. Moreover, $H_{2 t+1}(x, y)$ is the best possible lower (or upper) Hölder mean bound for $L_{t}(x, y)$ in (2.1) (or (2.2)).

\section{Proofs of main results}

Proof of Theorem 1.6 Since

$$
L_{0}(x, y)=A(x, y)=H_{1}(x, y), \quad L_{-1 / 2}(x, y)=G(x, y)=H_{0}(x, y)
$$

and

$$
L_{-1}(x, y)=H(x, y)=H_{-1}(x, y) .
$$


Therefore, Theorem 1.4 and (1.4) show that $\{(p, q) \mid(-1,-1),(0,0),(1,1)\} \subset D$, namely, for $\lambda=0,-1 / 2$ or -1 , the inequality

$$
\mathcal{K}\left(L_{\lambda}(x, y)\right)<L_{\lambda}(\mathcal{K}(x), \mathcal{K}(y))
$$

holds for all $x, y \in(0,1)$ with $x \neq y$. Thus $\mathcal{K}(r)$ is strictly $L_{\lambda, \lambda}$-convex on $(0,1)$ for $\lambda=0,-1 / 2$ and -1 .

Next, we divide the proof into four cases.

Case 1. $-1 / 2<\lambda<0$. Then it follows from Theorem 1.4 and (1.4) that $(2 \lambda+1,0) \in D$. Making use of Lemma 2.1 together with the monotonicity of $\mathcal{K}(r)$ on $(0,1)$, one has

$$
\mathcal{K}\left(L_{\lambda}(x, y)\right)<\mathcal{K}\left(H_{2 \lambda+1}(x, y)\right)<H_{0}(\mathcal{K}(x), \mathcal{K}(y))<L_{\lambda}(\mathcal{K}(x), \mathcal{K}(y))
$$

for all $x, y \in(0,1)$ with $x \neq y$.

Therefore, $\mathcal{K}(r)$ is strictly $L_{\lambda, \lambda}$-convex on $(0,1)$ for $-1 / 2<\lambda<0$.

Case 2. $-1<\lambda<-1 / 2$. Similarly, by Theorem 1.4 and Lemma 2.1 we have $(0,2 \lambda+1) \in D$ and

$$
\mathcal{K}\left(L_{\lambda}(x, y)\right)<\mathcal{K}\left(H_{0}(x, y)\right)<H_{2 \lambda+1}(\mathcal{K}(x), \mathcal{K}(y))<L_{\lambda}(\mathcal{K}(x), \mathcal{K}(y))
$$

for all $x, y \in(0,1)$ with $x \neq y$. This implies that $\mathcal{K}(r)$ is strictly $L_{\lambda, \lambda}$-convex on $(0,1)$ for $-1<\lambda<-1 / 2$.

Case 3. $\lambda>0$. For any $0<y<1$, letting $x \rightarrow 0^{+}$, then we have

$$
\begin{aligned}
\mathcal{K}\left(L_{\lambda}(x, y)\right)-L_{\lambda}(\mathcal{K}(x), \mathcal{K}(y)) & =\mathcal{K}\left(\frac{x^{\lambda+1}+y^{\lambda+1}}{x^{\lambda}+y^{\lambda}}\right)-\frac{\mathcal{K}^{\lambda+1}(x)+\mathcal{K}^{\lambda+1}(y)}{\mathcal{K}^{\lambda}(x)+\mathcal{K}^{\lambda}(y)} \\
& \rightarrow \frac{(\pi / 2)^{\lambda}[\mathcal{K}(y)-\pi / 2]}{(\pi / 2)^{\lambda}+\mathcal{K}^{\lambda}(y)}>0 .
\end{aligned}
$$

It follows from (3.1) that there exists $x_{0}=x(y) \in(0,1)$ such that $\mathcal{K}\left(L_{\lambda}(x, y)\right)>L_{\lambda}(\mathcal{K}(x)$, $\mathcal{K}(y))$ for all $x \in\left(0, x_{0}\right)$. Thus $\mathcal{K}(r)$ is not $L_{\lambda, \lambda}$-convex on $(0,1)$ for $\lambda>0$.

Case 4. $\lambda<-1$. For $0<x<1$, letting $y \rightarrow 1^{-}$, then one has

$$
\mathcal{K}\left(L_{\lambda}(x, y)\right)-L_{\lambda}(\mathcal{K}(x), \mathcal{K}(y)) \rightarrow \mathcal{K}\left(\frac{1+x^{\lambda+1}}{1+x^{\lambda}}\right)-\mathcal{K}(x)>0
$$

where we unitize $\left(1+x^{\lambda+1}\right) /\left(1+x^{\lambda}\right)>x$.

Making use of the analogous arguments in Case 3 we conclude that $\mathcal{K}(r)$ is not $L_{\lambda, \lambda}$ convex on $(0,1)$ for $\lambda<-1$.

Proof of Theorem 1.7 Clearly, by Theorem 1.5 we know that

$$
\{(p, q) \mid(-1,-1),(0,0),(1,1)\} \subset D^{*} .
$$

Thus, $\mathcal{E}(r)$ is strictly $L_{\lambda, \lambda}$-concave on $(0,1)$ for $\lambda=0,-1 / 2$ or -1 .

Next, we divide the proof into three cases. 
Case I. $-1 / 2<\lambda<0$ or $\lambda<-1$. Then it is easy to check that $(2 \lambda+1,2 \lambda+1) \in D^{*}$. Thus Theorem 1.5 and Lemma 2.1 together with the monotonicity of $\mathcal{E}(r)$ lead to the conclusion that

$$
\mathcal{E}\left(L_{\lambda}(x, y)\right)>\mathcal{E}\left(H_{2 \lambda+1}(x, y)\right)>H_{2 \lambda+1}(\mathcal{E}(x), \mathcal{E}(y))>L_{\lambda}(\mathcal{E}(x), \mathcal{E}(y))
$$

for all $x, y \in(0,1)$ with $x \neq y$.

Therefore, $\mathcal{E}(r)$ is strictly $L_{\lambda, \lambda}$-concave on $(0,1)$ for $-1 / 2<\lambda<0$ or $\lambda<-1$.

Case II. $-1<\lambda<-1 / 2$. Then by Theorem 1.5 and Lemma 2.1 we get

$$
\mathcal{E}\left(L_{\lambda}(x, y)\right)>\mathcal{E}\left(H_{0}(x, y)\right)>H_{0}(\mathcal{E}(x), \mathcal{E}(y))>L_{\lambda}(\mathcal{E}(x), \mathcal{E}(y))
$$

for all $x, y \in(0,1)$ with $x \neq y$.

Therefore, $\mathcal{E}(r)$ is strictly $L_{\lambda, \lambda}$-concave on $(0,1)$ for $-1<\lambda<-1 / 2$.

Case III. $\lambda>0$. For any $0<y<1$, letting $x \rightarrow 0^{+}$, then we have

$$
\begin{aligned}
\mathcal{E}\left(L_{\lambda}(x, y)\right)-L_{\lambda}(\mathcal{E}(x), \mathcal{E}(y)) & =\mathcal{E}\left(\frac{x^{\lambda+1}+y^{\lambda+1}}{x^{\lambda}+y^{\lambda}}\right)-\frac{\mathcal{E}^{\lambda+1}(x)+\mathcal{E}^{\lambda+1}(y)}{\mathcal{E}^{\lambda}(x)+\mathcal{E}^{\lambda}(y)} \\
& \rightarrow \frac{(\pi / 2)^{\lambda}[\mathcal{E}(y)-\pi / 2]}{(\pi / 2)^{\lambda}+\mathcal{E}^{\lambda}(y)}<0 .
\end{aligned}
$$

It follows from (3.2) that there exists $x_{1}=x(y) \in(0,1)$ such that $\mathcal{E}\left(L_{\lambda}(x, y)\right)<L_{\lambda}(\mathcal{E}(x), \mathcal{E}(y))$ for all $x \in\left(0, x_{1}\right)$. Thus $\mathcal{E}(r)$ is not $L_{\lambda, \lambda}$-concave on $(0,1)$ for $\lambda>0$.

\section{Competing interests}

The authors declare that they have no competing interests.

\section{Authors' contributions}

All authors contributed equally to the writing of this paper. All authors read and approved the final manuscript.

\section{Author details}

'School of Mathematics and Computation Sciences, Hunan City University, Yiyang, 413000, China. ${ }^{2}$ Department of Mathematics, Hangzhou Normal University, Hangzhou, 310036, China.

\section{Acknowledgements}

The authors wish to thank the anonymous referees for their careful reading of the manuscript and their fruitful comments and suggestions. The research was supported by the Natural Science Foundation of China under Grants 11301127 , 61374086 and 11171307, and the Natural Science Foundation of Zhejiang Province under Grant LY13A010004.

Received: 7 September 2015 Accepted: 1 December 2015 Published online: 15 December 2015

\section{References}

1. Bowman, F: Introduction to Elliptic Functions with Applications. Dover, New York (1961)

2. Byrd, PF, Friedman, MD: Handbook of Elliptic Integrals for Engineers and Scientists. Springer, New York (1971)

3. Anderson, GD, Qiu, S-L, Vamanamurthy, MK: Elliptic integral inequalities, with applications. Constr. Approx. 14(2), 195-207 (1998)

4. Anderson, GD, Vamanamurthy, MK, Vuorinen, M: Conformal Invariants, Inequalities, and Quasiconformal Maps. Wiley, New York (1997)

5. Anderson, GD, Vamanamurthy, MK, Vuorinen, M: Topics in Special Functions, Papers on Analysis. Rep. Univ. Jyväskylä Dep. Math. Stat., vol. 83, pp. 5-26. University Jyväskylä, Jyväskylä (2001)

6. Barnard, RW, Pearce, K, Schovanec, L: Inequalities for the perimeter of an ellipse. J. Math. Anal. Appl. 260(2), 295-306 (2001)

7. Borwein, JM, Borwein, PB: Pi and the AGM. Wiley, New York (1987)

8. Carlson, BC: Special Functions of Applied Mathematics. Academic Press, New York (1977)

9. Olver, FWJ, Lozier, DW, Boisvert, RF, Clark, CW: NIST Handbook of Mathematical Functions. Cambridge University Press, Cambridge (2010)

10. Kühnau, R: Eine Methode, die Positivität einer Funktion zu prüfen. Z. Angew. Math. Mech. 74(2), 140-143 (1994)

11. Alzer, H, Qiu, S-L: Monotonicity theorems and inequalities for the complete elliptic integrals. J. Comput. Appl. Math. 172(2), 289-312 (2004) 
12. Anderson, GD, Vamanamurthy, MK, Vuorinen, M: Functional inequalities for complete elliptic integrals and their ratios. SIAM J. Math. Anal. 21(2), 536-549 (1990)

13. Anderson, GD, Vamanamurthy, MK, Vuorinen, M: Functional inequalities for hypergeometric functions and complete elliptic integrals. SIAM J. Math. Anal. 23(2), 512-524 (1992)

14. Anderson, GD, Vamanamurthy, MK, Vuorinen, M: Generalized convexity and inequalities. J. Math. Anal. Appl. 355(2), 1294-1308 (2007)

15. András, S, Baricz, Á: Bounds for complete elliptic integral of the first kind. Expo. Math. 28(4), 357-364 (2010)

16. Barnard, RW, Pearce, K, Richards, KC: A monotonicity property involving ${ }_{3} F_{2}$ and comparisons of the classical approximations of elliptical arc length. SIAM J. Math. Anal. 32(2), 403-419 (2000)

17. Barnard, RW, Pearce, K, Richards, KC: An inequality involving the generalized hypergeometric function and the arc length of an ellipse. SIAM J. Math. Anal. 31(3), 693-699 (2000)

18. Chu, Y-M, Wang, M-K: Optimal Lehmer mean bounds for the Toader mean. Results Math. 61(3-4), 223-229 (2012)

19. Qiu, S-L, Vamanamurthy, MK: Sharp estimates for complete elliptic integrals. SIAM J. Math. Anal. 27(3), 823-834 (1996)

20. Qiu, S-L, Vamanamurthy, MK, Vuorinen, M: Some inequalities for the growth of elliptic integrals. SIAM J. Math. Anal. 29(5), 1224-1237 (1998)

21. Toader, G: Some mean values related to the arithmetic-geometric mean. J. Math. Anal. Appl. 218(2), 358-368 (1998)

22. Vamanamurthy, MK, Vuorinen, M: Inequalities for means. J. Math. Anal. Appl. 183(1), 155-166 (1994)

23. Wang, M-K, Chu, Y-M, Qiu, S-L, Jiang, Y-P: Bounds for the perimeter of an ellipse. J. Approx. Theory 164(7), 928-937 (2012)

24. Anderson, GD, Qiu, S-L, Vamanamurthy, MK, Vuorinen, M: Generalized elliptic integrals and modular equations. Pac. J. Math. 192(1), 1-37 (2000)

25. Baricz, A: Turán type inequalities for generalized complete elliptic integrals. Math. Z. 256(4), 895-911 (2007)

26. Heikkala, V, Lindén, $H$, Vamanamurthy, MK, Vuorinen, $M$ : Generalized elliptic integrals and the Legendre $\mathcal{M}$-function. J. Math. Anal. Appl. 388(1), 223-243 (2008)

27. Heikkala, V, Vamanamurthy, MK, Vuorinen, M: Generalized elliptic integrals. Comput. Methods Funct. Theory 9(1), 75-109 (2009)

28. Wang, M-K, Chu, Y-M, Qiu, S-L: Sharp bounds for generalized elliptic integrals of the first kind. J. Math. Anal. Appl. $429(2), 744-757(2015)$

29. Zhang, X-H, Wang, G-D, Chu, Y-M: Remark on generalized elliptic integrals. Proc. R. Soc. Edinb. A 139(2), 417-426 (2009)

30. Baricz, Á: Convexity of the zero-balanced Gaussian hypergeometric functions with respect to Hölder means. JPAM. J. Inequal. Pure Appl. Math. 8(2), Article ID 40 (2007)

31. Baricz, Á: Geometrically concave univariate distributions. J. Math. Anal. Appl. 363(1), 182-196 (2010)

32. Borwein, D, Borwein, J, Fee, G, Girgensohn, R: Refined convexity and special cases of the Blaschke-Santalo inequality. Math. Inequal. Appl. 4(4), 631-638 (2001)

33. Chu, Y-M, Wang, M-K, Jiang, Y-P, Qiu, S-L: Concavity of the complete elliptic integrals of the second kind with respect to Hölder means. J. Math. Anal. Appl. 395(2), 637-642 (2012)

34. Matkowski, J, Rätz, J: Convexity of power functions with respect to symmetric homogeneous means. Int. Ser. Numer. Math. 123, 231-247 (1997)

35. Matkowski, J, Rätz, J: Convexity with respect to an arbitrary mean. Int. Ser. Numer. Math. 123, 249-258 (1997)

36. Wang, G-D, Zhang, X-H, Chu, Y-M: A Hölder mean inequality for the Hersch-Pfluger distortion function. Sci. China Math. 40, 783-786 (2010) (in Chinese)

37. Wang, G-D, Zhang, X-H, Jiang, Y-P: Concavity with respect to Hölder means involving the generalized Grötzsch function. J. Math. Anal. Appl. 379(1), 200-204 (2011)

38. Wang, M-K, Chu, Y-M, Qiu, S-L, Jiang, Y-P: Convexity of the complete elliptic integrals of the first kind with respect to Hölder means. J. Math. Anal. Appl. 388(2), 1141-1146 (2012)

39. Zhang, X-H, Wang, G-D, Chu, Y-M: Convexity with respect to Hölder mean involving zero-balanced hypergeometric functions. J. Math. Anal. Appl. 353(1), 256-259 (2009)

40. Zhou, L-M, Qiu, S-L, Wang, F: Inequalities for the generalized elliptic integrals with respect to Hölder means. J. Math. Anal. Appl. 386(2), 641-646 (2012)

41. Chu, Y-M, Qiu, Y-F, Wang, M-K: Hölder mean inequalities for the complete elliptic integrals. Integral Transforms Spec. Funct. 23(7), 521-527 (2012)

42. Liu, Z: Remark on inequalities between Hölder and Lehmer means. J. Math. Anal. Appl. 247(1), 309-313 (2000)

\section{Submit your manuscript to a SpringerOpen ${ }^{\circ}$ journal and benefit from:}

- Convenient online submission

Rigorous peer review

- Immediate publication on acceptance

- Open access: articles freely available online

- High visibility within the field

- Retaining the copyright to your article 